\title{
Top-10 Tips for Getting Started with Magnetic Resonance Neurography
}

\author{
Majid Chalian, MD ${ }^{1}$ Avneesh Chhabra, MD ${ }^{1}$ \\ ${ }^{1}$ Musculoskeletal Imaging Division, Department of Radiology, \\ University of Texas, UT Southwestern Medical Center, Dallas, Texas \\ Semin Musculoskelet Radiol 2019;23:347-360.

\begin{abstract}
Address for correspondence Avneesh Chhabra, MD, Musculoskeletal Radiology, UT Southwestern Medical Center, 5323 Harry Hines Blvd, Dallas, TX 75390-9178

(e-mail: avneesh.chhabra@utsouthwestern.edu).
\end{abstract}

\author{
Abstract \\ Keywords \\ - magnetic resonance \\ neurography \\ - MRN \\ - peripheral nerve MRI \\ - neuropathy \\ - structured \\ interpretation
}

Magnetic resonance neurography (MRN), also known as MR neurography, is a dedicated imaging technique for the peripheral nerves, used both in a clinical setting and research. However, like any other new diagnostic processes, there are technical, cost, and patient selection issues to overcome as well as potential imaging pitfalls to recognize before MRN can be adopted efficiently into routine clinical practice. This review focuses on the 10 most important practical tips to get started with MRN with a view to shortening the time needed for radiologists to implement this clinically useful technique into their imaging practices.
Magnetic resonance neurography (MRN), or MR neurography, is a dedicated magnetic resonance imaging (MRI) technique that uses state-of-the-art pulse sequences and imaging protocols to visualize the peripheral nerves. First introduced by Filler et al, ${ }^{1}$ MRN techniques have considerably evolved over the last decade with the introduction of three-dimensional (3D) imaging pulse sequences and improvement in diffusion MR techniques.,3

Peripheral nerves are traditionally evaluated using a combination of clinical findings and electrodiagnostic studies such as electromyography (EMG) and nerve conduction studies (NCS). Although these techniques can confirm the presence of neuropathy and approximate the site of nerve pathology, they are operator and subject dependent, minimally invasive, and uncomfortable. They also do not define the structural cause of neuropathy or precisely localize the site of the neuropathy. ${ }^{4,5}$ In addition, electrodiagnostic tests can be normal up to 14 to 21 days after mild nerve injury. ${ }^{6-8}$

Ultrasound (US) is also used for the diagnosis of common nerve entrapment syndromes and neuropathies as well as for guiding treatment intervention. ${ }^{9-11}$ US is operator dependent and unable to show the subtle changes in neural composition seen by MRN, especially in mild neuropathy. US is also limited in the assessment of deeper anatomical locations and postoperative cases due to poor contrast resolution when echogenic perineural fat is obliterated. It also does not readily show changes in muscle composition induced by acute or subacute denervation. ${ }^{12}$

The small caliber of peripheral nerves as well as the presence of adjacent vascular structures of similar intensity limits the assessment of peripheral nerves if routine MR imaging is used. State-of-the-art advances in MR technology including dedicated multichannel radiofrequency surface coils have made it feasible to obtain high-resolution, highcontrast MRN imaging to delineate the small peripheral nerves, their abnormalities, and related features.

MRN was shown to be a useful adjunct to clinical electrodiagnostic (EMG/NCS) assessment. ${ }^{13,14}$ In addition, it can allow direct visualization of a neuropathy, more precise anatomical localization of the nerve abnormality and its cause (-Fig. 1), grading of the nerve injury, and depiction of muscle denervation. Consequently, MRN is now considered probably the best way to image peripheral neuropathy and is becoming more widely used in nonspecialized centers. ${ }^{15}$

Nevertheless, introducing MRN imaging into an MR clinical practice can be challenging. Education of patients and referring clinicians is essential in terms of expectations. Acute nerve injuries as seen commonly in trauma centers will have a different imaging approach than chronic neuropathies as seen more commonly in rehabilitation centers and pain control clinics. ${ }^{15}$
Issue Theme Top Ten Tips for MSK Imaging; Guest Editor, James F. Griffith, MB BCh, MRCP, FRCR, MD, FHKCR, FHKAM (Radiology)
Copyright (C) 2019 by Thieme Medical Publishers, Inc., 333 Seventh Avenue, New York, NY 10001, USA. Tel: +1(212) 584-4662.
DOI https://doi.org/ 10.1055/s-0039-1677727. ISSN 1089-7860. 

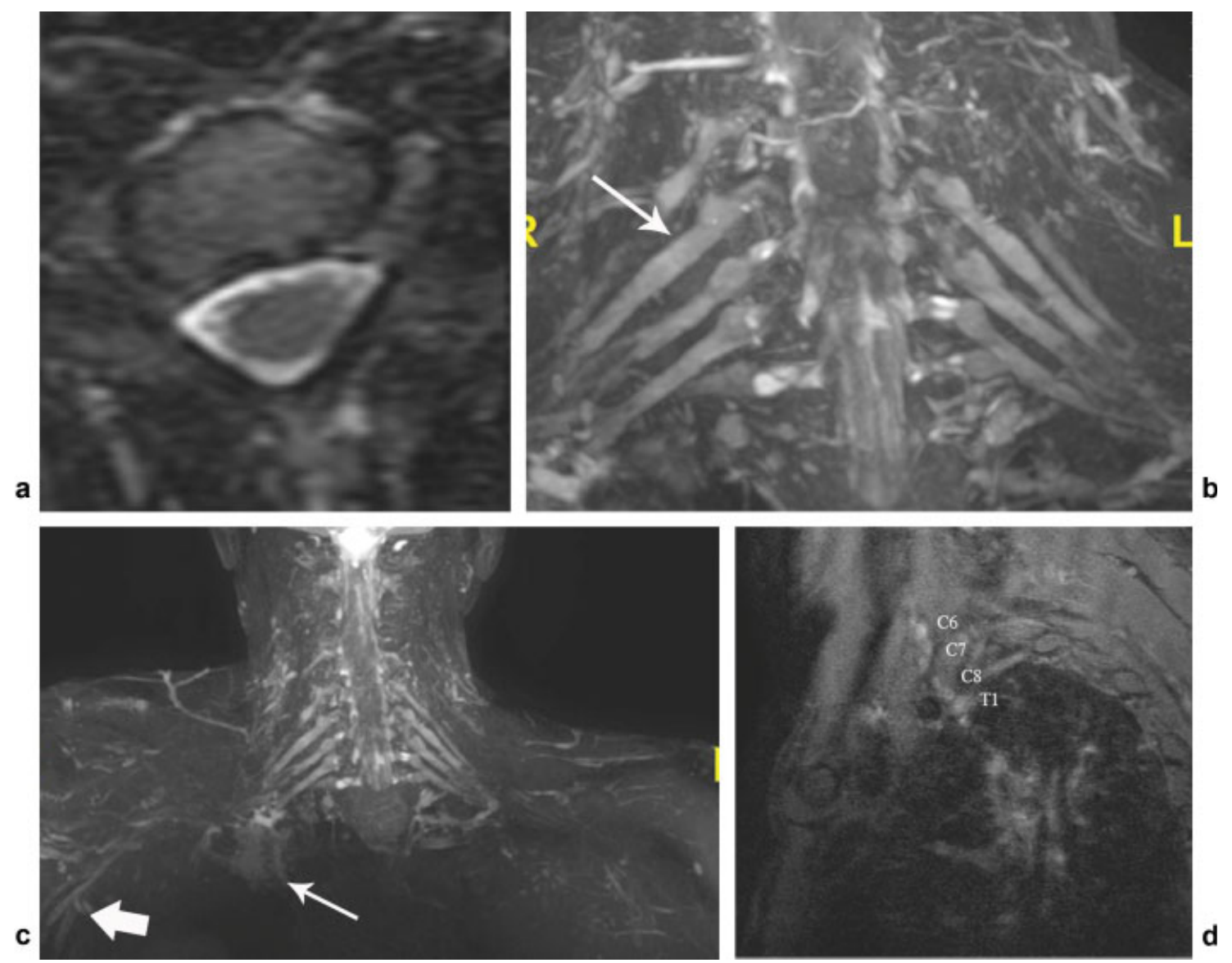

Fig. 1 A 60-year-old man with a motor vehicle accident some years previously presented with symptoms of right C6 neuropathy. (a) Axial T2 3D turbo spin-echo image shows moderate to severe right neural foraminal stenosis at the C6-C7 level. (b) Small field-of-view coronal 3D 18-mm slab maximum intensity projection (MIP) short tau inversion recovery (STIR) image depicts asymmetric thickening of right C6 preganglionic and postganglionic segments (arrow). (c) An unexpected mass (thin arrow) was found at the right lung apex on large field-of-view coronal MIP STIR imaging. There is traction of the right lower brachial plexus nerve roots by desmoplastic reaction of the apical lung mass. The nerves in the axillary pouch are bright and thickened (thick arrow). (d) Right sagittal STIR image demonstrates a thickened and bright C6 nerve root, normal appearance of the $\mathrm{C} 7$ and $\mathrm{C} 8$ nerve roots, and bright $\mathrm{T} 1$ nerve root due to desmoplastic reaction by the right lung apical mass. The final diagnosis was Pancoast tumor invading the inferior brachial plexus.

MRN should be approached as a team task for both radiologists and clinicians because there is learning curve on both sides. Radiologists need to know clinicians' expectations of imaging, terminology, clinical pathologies, and operative approaches. Clinicians should be aware of the clinical indications for MRN, as well as the limitations and pitfalls of the technique. Multidisciplinary conferences or case discussions are beneficial in this regard and can potentially prevent unnecessary imaging and miscommunication. This review focuses on the 10 practical tips considered most useful for radiologists wishing to get started with MRN, focusing on the technical challenges, image acquisition, the common interpretation pearls, and writing a clinically relevant report.

\section{Tip 1: Acquire a Good Knowledge of Neuromuscular Anatomy}

The nervous system encompasses the central nervous system (CNS), made up of the brain, spinal cord, and retina, and the peripheral nervous system (PNS). The PNS comprises all other neural structures including nerves, ganglia, receptors, and motor nerve endings. All PNS structures are crucial in transmitting to and from the CNS. Nerves are the main focus of PNS imaging due to their length, function, and predisposition to pathologic entities. ${ }^{12}$

A peripheral nerve has a unique structure that enables it to conduct nerve stimuli. The main component is the nerve axon, an elongated extension of the nerve body specialized for electrical impulse conduction. The presence of supporting Schwann cells that cover the axons differentiate nerves into myelinated or unmyelinated fibers. The myelinated nature of a nerve fiber determines the nerve conduction velocity. Myelinated and unmyelinated nerve fibers lie in a connective tissue called the endoneurium, comprising a collagen matrix, fibroblasts, and fine blood capillaries. Multiple nerve fibers and their surrounding endoneurium create nerve fascicles encased by a concentric supporting cell layer, called the perineurium. Multiple nerve fascicles (ranging from 1 to 3 in small peripheral sensory nerves to 250 in large more centrally located nerves such as the sciatic nerve) are grouped by a dense connective tissue, the epineurium. The epineurium has outer and inner layers. The outer epineurium is made up of a denser 
connective tissue; the inner epineurium contains adipose tissue and blood vessels called vasa nervorum. ${ }^{16,17}$ Intraneural fat and vessels become more prominent with aging. ${ }^{8}$ In addition, asymptomatic muscle atrophy and fatty infiltration are seen with aging, not to be confused with chronic denervation. ${ }^{18}$ Nerves can be bifid and can demonstrate an intramuscular course as a normal variant. ${ }^{19}$

From a functional standpoint, peripheral nerves are divided into sensory, motor, and mixed nerves. There is usually a predictable internal topographic distribution of nerve fascicles within mixed peripheral nerves. For instance, the ulnar nerve at the elbow contains sensory, motor, and cutaneous fascicles, distributed from its lateral to medial side. ${ }^{20}$ Knowledge of peripheral nerve locations, the common sites of nerve entrapment, common normal variants, and aberrant nerve courses will help the radiologist avoid misinterpretation. Radiologists should stay abreast with new knowledge from scientific journal articles and online teaching aids, especially early on after implementation of a clinical MRN practice. Use of anatomy atlases in similar planes to MRN imaging increases the reader's ability to find abnormalities as well as normal variants, such as atypical nerve courses.

\section{Tip 2: Understand the Pathophysiology and Classification of Neuropathies}

Nerve lesions may be divided into traumatic, inflammatory, infectious, hereditary, radiation-induced, neoplastic, and neoplastic-like entities. ${ }^{21}$ Following nerve injury, the axon and the supporting Schwann cells begin to disintegrate within a few hours. This process, called Wallerian degeneration, starts immediately distal to the site of injury and extends distally. Wallerian degeneration is due to the interruption of axoplasmic flow that delivers substances essential to nerve survival.

There are different classifications for peripheral nerve injuries. One of the most frequently used grading systems, which shows moderate to good correlation with MRN findings, ${ }^{22}$ is the Seddon and Sunderland classification, introduced by Seddon ${ }^{23}$ in 1943 and modified by Sunderland ${ }^{24}$ in 1951 (-Table 1).

- Sunderland class I (neuropraxia according to Seddon) usually follows a moderate compression injury leading to segmental demyelination and focal conduction block although otherwise no axonal injury. Recovery is full and seen within a few days to 2 months of injury. There is no Tinel's sign; EMG is normal but interference potential can be decreased. On MRI, there are no signs of Wallerian degeneration including muscle denervation, although the affected nerve demonstrates mild increased T2 signal intensity (SI) on MRN.

- Sunderland class II (axonotmesis according to Seddon) usually follows a crush or severe compression injury causing segmental demyelination and axonal injury, although the endoneurium, perineurium, and epineurium are preserved. Recovery is complete but slower than with Sunderland class I, taking 2 to 4 months. There is complete loss of nerve function distally leading to Wallerian degeneration, a positive Tinel's sign, an abnormal EMG, and muscle denervation.

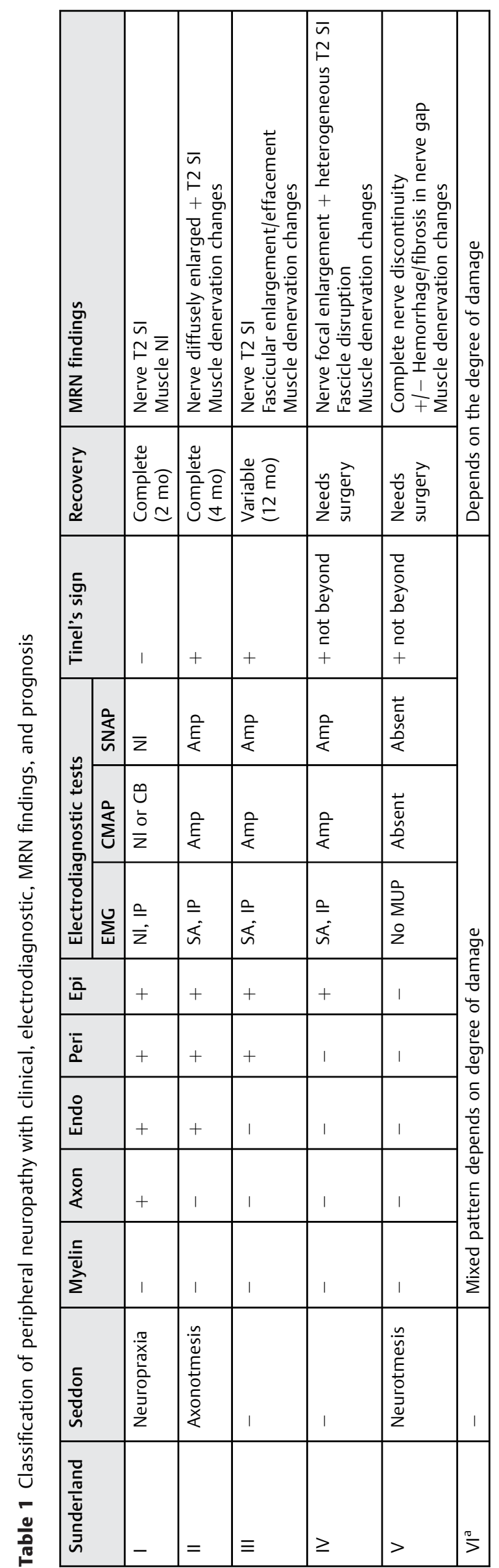


On MRI, there is diffuse nerve enlargement $(<50 \%$ increase in nerve thickness) with increased nerve T2 SI and appreciable muscle denervation changes.

- Sunderland class III usually follows a severe crush or transection injury creating segmental demyelination, axonal injury, and disruption of the endoneurium with preservation of the perineurium and epineurium. Recovery is variable and could be complete but takes time, with a typical pace of $1 \mathrm{~mm} /$ day for nerve regeneration. Nerve function, EMG, and Tinel's sign are the same as for Sunderland class II. On MRN, diffuse fascicular enlargement/ effacement within the nerve due to edema is seen, as well as muscle denervation changes. There is $>50 \%$ increase in nerve thickness as well as more fascicular edema/atrophy compared with Sunderland class II.

- Sunderland class IV usually follows a transection injury resulting in segmental demyelination, axonal injury, and disruption of the endoneurium and perineurium although with preservation of the epineurium. Therefore, no visible nerve discontinuity is seen. Recovery is unlikely without surgical intervention such as direct suturing or nerve grafting following neuroma resection. Nerve function and EMG are the same as Sunderland class II/III. Tinel's sign is present but not beyond the level of the injury. MRN shows focal nerve enlargement with heterogeneous T2 SI and fascicular disruption as well as muscle denervation changes. Hemorrhage and fibrous tissue entangle the regenerating neural islands inhibiting distal axonal growth and leading to formation of a neuroma-in-continuity (NIC).

- Sunderland class V (neurotmesis according to Seddon) usually follows a transection injury with complete disruption of all the nerve tissues including the axons, endoneurium, perineurium, and epineurium. There is complete nerve discontinuity with a gap that may be filled by hemorrhage or fibrous tissue. There will be no recovery without surgical intervention. EMG fails to show a motor unit potential. Tinel's sign is positive but not beyond the level of injury as in Sunderland class IV. MRN depicts complete nerve discontinuity with a fluid gap or possible fibrosis/hemorrhage filling the gap and muscle denervation. An end-bulb neuroma might be present.

Later on, Mackinnon and Dellon ${ }^{25}$ described another class of nerve injury pattern (outside-in model) as an addition to the Seddon and Sunderland classification (inside-out model). This type of injury, often considered class VI, is a mixed injury involving variable tissue injury across the transverse section of the nerve. Clinical, electrodiagnostic, and MRN findings as well as recovery rate are variable depending on the tissues most damaged. This injury usually results from direct nerve injury due to penetrating trauma or fracture/dislocation.

Based on clinical and electrodiagnostic tests, it is:

1. Difficult to detect mild nerve damage (neuropraxia).

2. Electrical responses can be normal despite complete nerve transection up to a week after insult. ${ }^{26}$

3. Electrodiagnostic tests do not clearly differentiate between higher grades of nerve injury (class II-IV).
Understanding the degree of neural tissue injury is crucial to estimating recovery potential and need for surgery. For instance, class II nerve injury is usually treated medically, whereas class IV or V nerve injury needs surgical intervention. Early diagnosis is important because a delay leads to muscle atrophy, slow rehabilitation, and poor functional recovery. ${ }^{27}$ Currently, serial electrodiagnostic studies with or without surgical explorations are used to determine the severity of nerve injury. MRN helps minimize the time between injury, assessment, and treatment.

Familiarity with common disease entities helps derive the correct MRN diagnosis. These include predominant motor dysfunction neuropathies, such as multifocal motor neuropathy, amyotrophic lateral sclerosis, polio, and perineurioma; predominant sensory dysfunction neuropathies such as Sjögren's syndrome and sensory-type chronic demyelinating polyneuropathy; mixed motor/sensory dysfunction neuropathies such as radiculopathy and chronic demyelinating polyneuropathy; toxic/dying back neuropathies, such as Charcot-Marie-Tooth (CMT) disease and diabetes mellitus (DM); and autonomic dysfunction with peripheral neuropathy such as DM and amyloidosis.

\section{Tip 3: Use MRN for the Proper Clinical Indication}

Peripheral nerve pathologies can be broadly classified as systemic and local diseases. ${ }^{28} \mathrm{~A}$ myriad of systemic diseases affects the peripheral nerves. These can be categorized as vasculitides; radiation-induced neuropathy/plexopathy; metabolic diseases such as DM, hyperlipidemia, and amyloidosis; neurocutaneous syndromes such as neurofibromatosis and schwannomatosis; infectious including viruses or mycobacterium leprae; hereditary diseases such as CMT; acute or chronic demyelinating conditions such as chronic inflammatory demyelinating polyneuropathy; and idiopathic diseases such as multifocal motor neuropathy. ${ }^{21,29}$ Systemic conditions produce a multifocal/multicompartmental neuropathy and are usually diagnosed clinically. Biochemical markers and electrodiagnostic tests are currently the most useful diagnostic tests. MRN in systemic polyneuropathy is used in cases when there is mass lesion or a worsening focal neuropathy raising concern of a primary or superimposed nerve entrapment. Sometimes, these systemic neuropathic conditions may be incidentally discovered if the patient is imaged for pain or internal joint derangement, and in this sense, it is also helpful to be aware of the imaging appearances and common patterns of diffuse neuropathy.

Local conditions affecting peripheral nerves include neuropathies due to trauma; compressive neuropathies, such as tunnel syndromes in various anatomical locations; traction neuropathies due to repetitive activities, bad footwear, ankle instability, and functional compartment syndromes; neoplastic conditions such as perineural tumors or peripheral nerve sheath tumors; and focal infections. ${ }^{8,29}$

As discussed earlier, nerve injuries were traditionally classified according to the Seddon and Sunderland systems (-Table 1). Compressive neuropathies are usually due to 
increased pressure within an anatomical tunnel. Increased pressure leads to venous congestion, hyperemia, blockade of axoplasmic flow, and finally proximal epineurial edema and distal Wallerian degeneration. That is why larger peripheral neural fascicles are first affected. Space-occupying lesions are a less frequent cause of compressive neuropathy. The mass can be intraneural involving the fascicles or perineural creating nerve entrapment, encasement, or displacement. The primary mechanism of traction neuropathy is shear force followed by ischemia.

For systematic conditions, MRN is used as a complementary or problem-solving technique, whereas for local conditions, it serves as the primary investigative modality. In both conditions, MRN is performed without intravenous contrast unless there is a suspicion of tumor or infection.

\section{Tip 4: Use Appropriate Hardware and Software}

MRN reporting relies on seeing and assessing specific subtle morphological features of the peripheral nerves, such as SI alteration, fascicular size and morphology, and perineural fat plane obliteration or fibrosis. Therefore, imaging peripheral nerves benefits from the use of higher field magnets, dedicated high-resolution coils, updated pulse sequences with optimal image parameters, and occasionally the use of specific pulse sequences with lengthier acquisition times. ${ }^{29}$

Obtaining images with high signal-to-noise ratio (SNR) and the best possible resolution is desired, although it is technically challenging and often a major limiting factor in MRN. Hence the use of 3-T scanners is recommended. High-field scanners provide higher SNR and allow thin section (2-3.3 $\mathrm{mm}$ ) acquisition and higher matrix size leading to improved in-plane resolution. In addition, 3-T scanners allow use of parallel imaging that helps reduce acquisition time and, as a result, minimize motion and breathing artifacts. Compressed sense and partial Fourier techniques are also available on some newer scanners that helps reduce acquisition times. ${ }^{30}$

MRN studies have been performed on 1.5-T systems including the use of diffusion tensor imaging (DTI). ${ }^{30,31}$ However, a longer acquisition time is required to obtain good SNR, and image resolution may be degraded by motion due to longer scan time and possibly smaller matrix size. In addition, 3D imaging is often limited on $1.5 \mathrm{~T}$ with suboptimal fat suppression. The 3-T imaging allows longer T1 and shorter T2 relaxation times, and therefore 3-T protocols cannot be automatically transferred to 1.5 -T systems without modifying the imaging parameters including reducing bandwidth. We highly recommend using 3-T systems, whenever possible, for peripheral nerve imaging. ${ }^{32,33}$ The 1.5-T might be preferred over $3 \mathrm{~T}$ when there is metal in the immediate field of view (FOV).

To further increase SNR, dedicated high-resolution multichannel phased-array surface coils should be used even over a large FOV. Examples of best use cases include a 60-channel surface body coil for the lumbosacral plexus and pelvic nerves, 18- to 60-channel coil for the brachial plexus, 16-channel wrist coil for the median and ulnar nerves at the wrist, 16-channel ankle coil for the tarsal tunnel, and a 15-channel knee coil for the peroneal nerve.

Multichannel flexible coils are also useful for the extremities. For example, a 36-channel peripheral coil can be used to image the leg, and a 4- to 8-channel flexible coil can be used for the forearm. ${ }^{29,34}$ It is important to image the joint and nonjoint extremity separately with different FOVs and not to have $>30 \%$ blank air space around the extremity area being imaged. If imaging the whole body, coils should be wrapped close to the body and can be strapped to each other to avoid blank air space between different torso coils. The torso coils can be combined with the spine array coils to obtain uniform imaging of the lumbosacral plexus. For occipital nerve imaging or peripheral trigeminal nerve imaging in the face, we use a 32-channel head coil.

Available independent software that allows thick slab maximum intensity and oblique/curved planar reformat projections should be used for evaluation of the longitudinal nerve axes similar to angiography. In-line reconstruction using thin client tools is preferred for image interpretation, and images can be sent directly to the picture archiving and communication system (PACS) for consultation and future review.

\section{Tip 5: Follow the Correct Imaging Protocol}

Different institutions/radiologists have different approaches to how to protocol MRN studies. Some radiologists tailor patient-specific protocols based on the clinical question, body part to be imaged, and required FOV. We recommend implementing standard protocols for different body parts and, if necessary, for some specific disease entities, (e.g., for carpal tunnel, use a wrist MRN protocol; for forearm and hand pain, use a forearm and wrist MRN protocol; for chest wall pain, use an intercostal neuralgia protocol that includes imaging of the thoracic spine and $3 \mathrm{D}$ imaging of the chest wall).

This standardized approach has benefits for the interpreting radiologists, referring clinicians, MR technicians, and patients. Having preset protocols shortens the learning curve for radiologists and clinicians by having the same set of images for specific body parts. This helps everyone get used to the normal anatomy and become familiar with nerve appearances in certain planes. Standard imaging protocols are particularly important for less experienced MR technicians. From the patient's standpoint, standardized protocols allow them to have an estimate of acquisition time. An intranet-based in-house electronic ordering system in our institution enables clinicians to request specific study types from a predefined dropdown list (e.g., brachial plexus, lumbosacral plexus, sciatic nerve, wrist nerves, etc.).

The FOV should be adjusted to the area of interest and be as small as possible for small extremity nerves and as large as necessary to cover all nerves in the brachial or lumbosacral plexus. A small FOV may create wraparound artifact in the extremities, and therefore applying a certain percentage of phase oversampling is recommended. ${ }^{35}$ Peripheral nerves are elongated structures with some extending $>1 \mathrm{~m}$ in length such as the sciatic, tibial, or peroneal nerves. It is 
important to have sufficient coverage of the body area so as not to miss important pathologies.

In addition, it is often unclear clinically where a nerve injury is located, especially in cases of diffuse neuropathy or nonspecific signs/symptoms, and therefore imaging of the entire nerve course is often necessary. In fact, missing pathology due to limited nerve coverage is a common imaging pitfall that should be avoided. In these circumstances, it is recommended to increase axial slice thickness (up to 5-6 mm) while keeping high axial in-plane resolution. ${ }^{29}$ After screening a large area, small FOV imaging of an area of interest can then be performed to obtain multiplanar high-resolution MRN imaging.

Generally speaking, MRN relies on high-resolution twodimensional (2D) and isotropic 3D spin-echo (turbo spin-echo or fast spin-echo) sequences with T1- and T2-weighted contrast. The 2D T2-weighted sequences are the workhorse of MRN due to their wide availability, familiarity, and easy reproducibility. ${ }^{36,37}$ Spectral adiabatic inversion recovery (SPAIR) or Dixon-based fat suppression provides excellent fat suppression for these T2-weighted images. For the brachial plexus, sagittal plane short tau inversion recovery (STIR) images are useful because SPAIR fat suppression commonly fails along the curvatures for the neck and thoracic inlet.

Although high-resolution 2D images obtained in the perpendicular plane are usually sufficient for detecting and characterizing nerve lesions, corresponding axial T1weighted images outline the nerve anatomy and perineural fat planes; and respective axial diffusion images (especially high b-value, $600-1000 \mathrm{~s} / \mathrm{mm}^{2}$ ) render the nerve abnormality conspicuous (-Fig. 2). Isotropic 3D images demonstrate the nerve abnormality in its longitudinal axis in multiple arbitrary planes. Thick slab maximum intensity projection (MIP) reconstructions from these 3D images better emphasize the nerve pathologies (signal alterations, NIC, and endbulb neuroma). These MIP reconstructions are particularly helpful for surgical mapping as well as facilitating discussion during multidisciplinary conferences. ${ }^{29}$

In general, the echo time (TE) for 2D T2-weighted imaging is kept at $\sim 60$ to $65 \mathrm{~ms}$ and matrix $>256$ to obtain enough SNR to depict the nerve fascicles while minimizing the magic angle artifact. For 3D imaging, the TE time is kept at $\sim 78 \mathrm{~ms}$ with an isotropic voxel of 1.4 to $1.5 \mathrm{~mm}$ for imaging of the plexuses. $^{38}$ For extremities and facial nerve imaging, 3D reversed fast imaging in steady-state precession (PSIF) is used for selective nerve demonstration with a TE of $2.5 \mathrm{~ms}$ and an isotropic voxel size of $0.9 \mathrm{~mm}$. Steady-state imaging using $0.65-\mathrm{mm}$ isotropic voxels is helpful to depict intrathecal rootlets, and 3D STIR provides excellent imaging of the extrathecal segments (-Fig. 3). Suggested protocols for brachial plexus, lumbosacral plexus, and extremity MR neurography are outlined in - Tables $\mathbf{2}, \mathbf{3}$, and $\mathbf{4}$. If metal is present in the immediate FOV, 1.5-T imaging and various metal reduction strategies are used (e.g., low TE, high bandwidth, high echo train length, frequency encoding gradient along the metal axis, and high matrix and low echo spacing) and techniques such as the commercially available MARS, WARP, or OMAR techniques. ${ }^{39}$
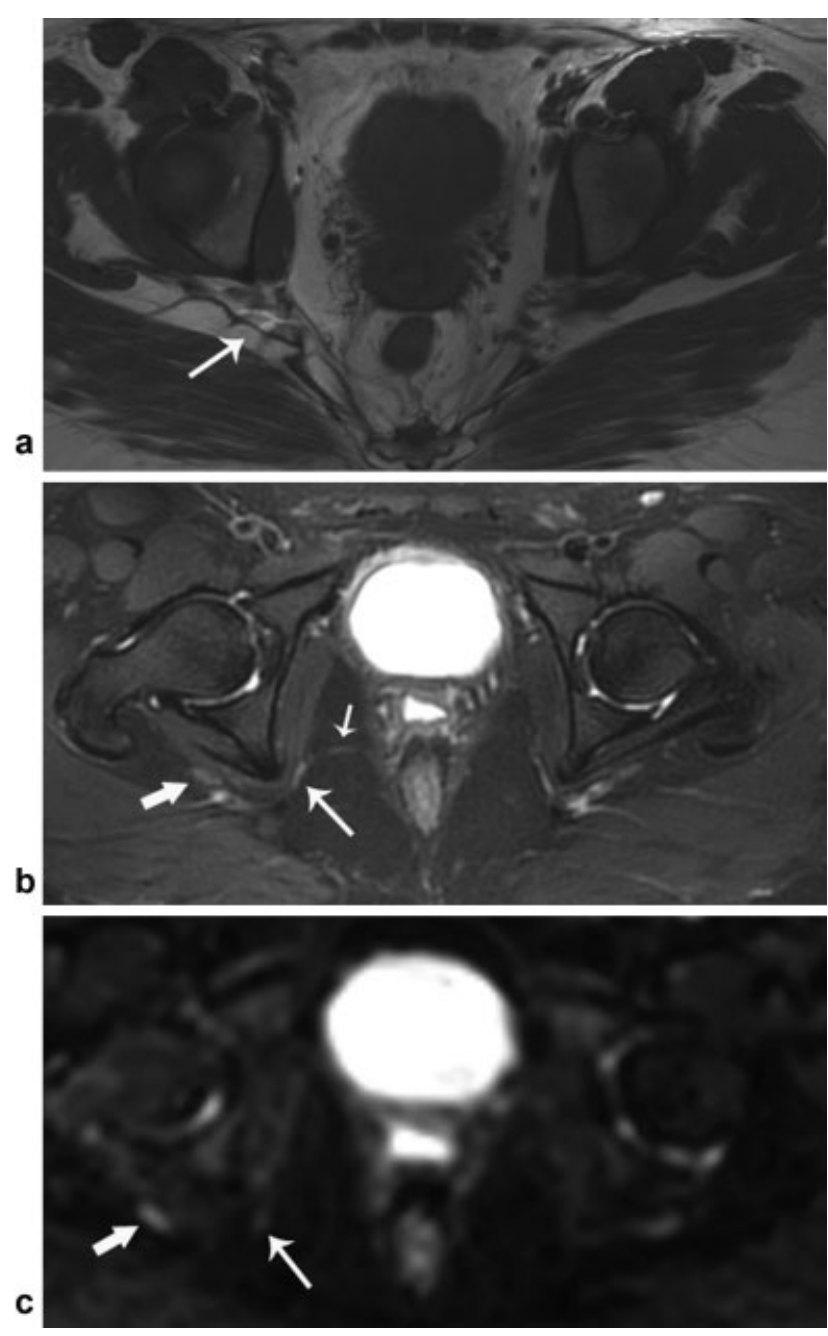

Fig. 2 A 41-year-old woman with history of a complicated vaginal delivery presented with pain and a swollen feeling in the perineum. (a) Axial T1-weighted image shows fibrotic bands and scar tissue along the right posterolateral pelvic wall in close proximity to the course of sciatic and pudendal nerves (arrow). (b) Axial T2 SPAIR image demonstrates an asymmetrically bright right sciatic nerve (thick arrow), right pudendal nerve (long thin arrow), and its inferior hemorrhoidal branch (small thin arrow). (c) Axial diffusion tensor imaging (b factor: $600 \mathrm{~s} / \mathrm{mm}^{2}$ ) confirms signal abnormality in the previously mentioned nerves correlating with the patient's symptoms and consistent with iatrogenic fibrotic entrapment neuropathy of the sciatic nerve (thick arrow) and pudendal nerve (thin arrow).

\section{Tip 6: Know How to Use Diffusion Imaging}

MRN protocols include more advanced imaging techniques such as diffusion-weighted imaging (DWI) including DTI and tractography. Using diffusion imaging along with MRN and tractography might be needed to get the most out of MRN studies. However, application of these techniques for small peripheral nerves can be technically challenging and difficult to implement. ${ }^{36,40}$

The surrounding fat and vessels are essentially suppressed in DWI, and this effective background suppression enhances the nerve conspicuity, helping to diagnose subtle nerve pathologies. $^{41,42}$ DWI provides quantitative parameter for nerve evaluation, such as apparent diffusion coefficient (ADC), and 

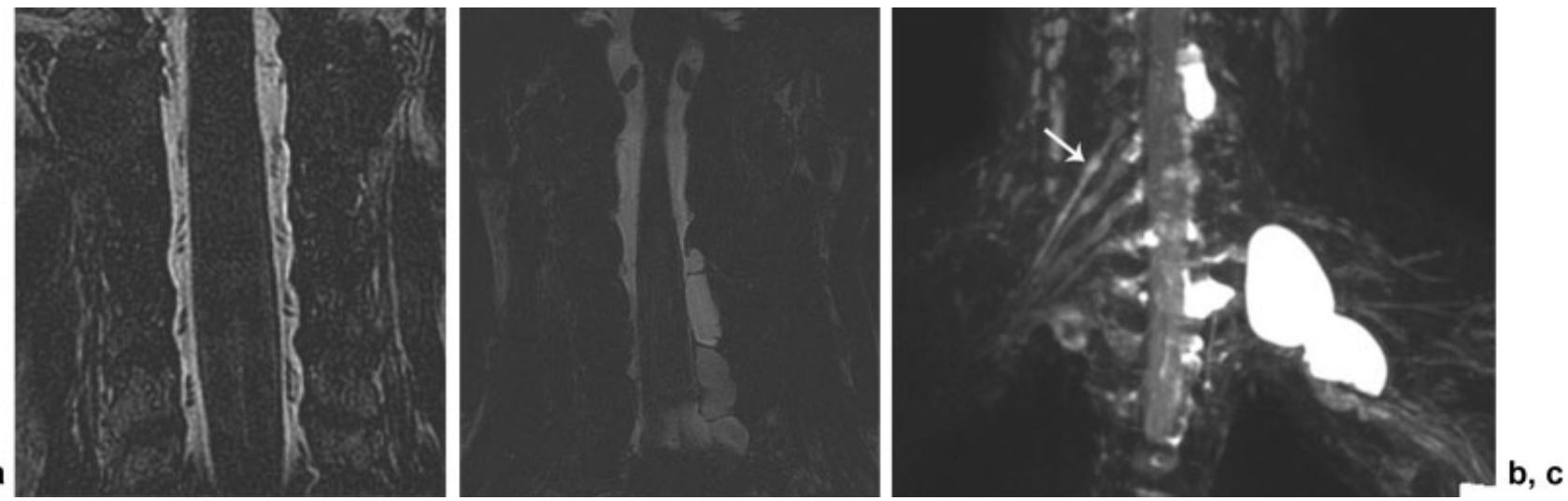

Fig. 3 (a) Multiplanar reconstruction (MPR) coronal oblique FIESTA image clearly depicts the intrathecal preganglionic nerve roots and is extremely helpful when evaluating suspected nerve root avulsion or other intrathecal pathologies. (b) MPR coronal oblique FIESTA image in a 21-year-old patient referred with left upper extremity weakness reveals extensive posttraumatic left-sided preganglionic nerve root avulsions from C4 through T2 with large pseudomeningoceles compatible with Sunderland class V nerve injury. (c) Coronal 3D 18-mm slab maximum intensity projection short tau inversion recovery image better demonstrates postganglionic extrathecal nerve roots and plexus. Note is made of bright postganglionic right C5 nerve root (arrow) consistent with a stretch injury (Sunderland class II/III).

Table 2 Brachial plexus 3-T MRN protocol

\begin{tabular}{|l|l|l|l|l|l|l|l|}
\hline Sequence & Plane & Matrix & $\begin{array}{l}\text { Gap, } \\
\%\end{array}$ & Voxel size, mm & TR & TE & Comment \\
\hline 3D STIR SPACE & Coronal & $400 \times 400$ & 0 & Isotropic 1.4-1.5 & $1,500-2,000$ & $75-80$ & $\begin{array}{l}\text { Cover both sides. Make 7- to } \\
10-m m \text { coronal MIP slabs. TI } \\
\text { of 230 ms }\end{array}$ \\
\hline 3D T2 SPACE & Sagittal & $320 \times 290$ & 0 & Isotropic 0.9 & $1,500-2,000$ & $110-120$ & $\begin{array}{l}\text { Cover neural foramina on both } \\
\text { sides }\end{array}$ \\
\hline $\begin{array}{l}\text { FIESTA/bFFE/ } \\
\text { TrueFISP }\end{array}$ & Coronal & $330 \times 330$ & 0 & Isotropic 0.6-0.65 & 6 & 4 & $\begin{array}{l}\text { Generate axial and sagittal } \\
\text { reconstructions }\end{array}$ \\
\hline 2D T1 TSE & Axial & $>256$ & 10 & 4 mm & 700 & $6-9$ & Cover both sides \\
\hline STIR right & Sagittal & $>256$ & 10 & $3.5-4.0$ & $1,500-2,000$ & $25-35$ & $\begin{array}{l}\text { Cover from left para-midline to } \\
\text { right shoulder }\end{array}$ \\
\hline STIR left & Sagittal & $>256$ & 10 & $3.5-4.0$ & $1,500-2,000$ & $25-35$ & $\begin{array}{l}\text { Cover from right para-midline to } \\
\text { left shoulder }\end{array}$ \\
\hline DTI & Axial & $128-192$ & 0 & 4.0 & 10,000 & $60-70$ & $\begin{array}{l}\text { b-values of } 50 \text { and } 800, \\
12 \text { directions. SPAIR fat saturation }\end{array}$ \\
\hline $\begin{array}{l}\text { 3D T1 mDixon } \\
\text { (optional) }\end{array}$ & Coronal & $340 \times 340$ & 0 & Isotropic 1.0-1.5 & $8-12$ & 4 & $\begin{array}{l}\text { Cover both sides. Pre- and } \\
\text { postcontrast if needed }\end{array}$ \\
\hline
\end{tabular}

Abbreviations: bFFE, balanced fast field echo; D, dimensional; DTI, diffusion tensor imaging; FIESTA, fast imaging employing steady-state acquisition; MIP, maximum intensity projection; SPACE, sampling perfection with application optimized contrasts using different flip angle evolution; STIR, short tau inversion recovery; TE, time of echo; TI, time of inversion; TR, time of repetition; TrueFISP, fast imaging with steady-state precession; TSE, turbo spin echo.

- On coronal 3D STIR SPACE, coverage is from base of skull to T3-T4 level. Side-to-side coverage is shoulder point to shoulder point and includes both sides.

- On sagittal T2 SPACE and coronal FIESTA, focus only on C spine including both neural foramina.

- Sagittal STIRs are critical to the examination, so repeat if there is pulsation artifact or motion present.

- For large subjects, increase voxel size to 1.5 to $2 \mathrm{~mm}$.

DTI in addition provides fractional anisotropy (FA) and tractography. When nerve pathology is present, ADC values increase, and FA values decrease. ${ }^{32,40-42}$ Evaluation of peripheral nerve microarchitecture and fascicles has been made feasible by DTI. Although some applications such as the ADC value measurement is easy to perform in routine clinical settings for tumors, application of DTI including tractography of the small peripheral nerves is still challenging and time consuming. It has the potential to allow differentiation of axonal versus demyelinating pathologies similar to electrophysiology but is currently mainly reserved for tumors and research purposes. ${ }^{29,43-46}$

\section{Tip 7: How to Interpret MRN Studies}

The normal peripheral nerve has a continuous course, uniform contour, and a diameter similar to the accompanying arteries or contralateral nerve. ${ }^{47}$ Normal peripheral nerves should have a smooth course without sharp angulation or deviation, or focal caliber change at nonbranching sites. This is diagnostically important for nerve entrapments where there is deviation in the normal neural course as well as compressive features upon the nerve. Irregular contour, abrupt change in caliber, or diffuse persistent increased 
Table 3 Lumbosacral plexus 3-T MRN protocol

\begin{tabular}{|c|c|c|c|c|c|c|c|}
\hline Sequence & Plane & Matrix & $\begin{array}{l}\text { Gap, } \\
\%\end{array}$ & Voxel size, $\mathrm{mm}$ & TR & TE & Comment \\
\hline 3D SHINKEI/STIR & Coronal & $460 \times 460$ & 0 & Isotropic 1.5 & 1,500 & $78-80$ & $\begin{array}{l}\text { Cover both sides. Make 8-mm- } \\
\text { thick MIP slabs }\end{array}$ \\
\hline 2D T2 TSE & Sagittal & $370 \times 300$ & 10 & $3.5-4.0$ & 3,500 & 45 & Focus on lumbar spine \\
\hline 2D T2 TSE & Axial & $300 \times 300$ & 10 & $3.5-4.0$ & 3,500 & 45 & Focus on lumbar spine \\
\hline 2D T1 TSE & Axial & $700 \times 600$ & 10 & 4.0 & 700 & $6-9$ & $\begin{array}{l}\text { Cover both sides from L1 to lesser } \\
\text { trochanter }\end{array}$ \\
\hline T2 SPAIR & Axial & $400 \times 400$ & 10 & 4.0 & 4,000 & $60-65$ & $\begin{array}{l}\text { Cover both sides from L1 to lesser } \\
\text { trochanter }\end{array}$ \\
\hline $\begin{array}{l}\text { 3D T1 Dixon } \\
\text { (optional) }\end{array}$ & Axial & $260 \times 260$ & 0 & Isotropic 1.0 & 12 & 5 & $\begin{array}{l}\text { Cover both sides from L1 to lesser } \\
\text { trochanter. Pre- and postcontrast } \\
\text { if needed }\end{array}$ \\
\hline DTI & Axial & $128 \times 192$ & 0 & $4-5$ & 10,000 & $60-70$ & $\begin{array}{l}\text { b-values of } 0 \text { and } 600,15 \text { direc- } \\
\text { tions. Cover from } L 3-L 4 \text { to lesser } \\
\text { trochanter. SPAIR- fat saturation }\end{array}$ \\
\hline STIR (optional) & Axial & $320 \times 320$ & 10 & 4.0 & 2,000 & $30-35$ & If fat saturation fails on SPAIR \\
\hline $\begin{array}{l}\text { FIESTA/bFFE/TrueFISP } \\
\text { (optional) }\end{array}$ & Sagittal & $340 \times 340$ & 0 & Isotropic $0.6-0.65$ & 12 & 4 & Coronal and axial reconstructions \\
\hline
\end{tabular}

Abbreviations: 3D SHINKEI, 3D nerve sheath signal increased with inked rest-tissue RARE imaging; bFFE, balanced fast field echo; D, dimensional; DTI, diffusion tensor imaging; FIESTA, fast imaging employing steady-state acquisition; MIP, maximum intensity projection; STIR, short tau inversion recovery; TE, time of echo; TR, time of repetition; TrueFISP, fast imaging with steady-state precession; TSE, turbo spin echo.

- On coronal SHINKEI, cover both sides from skin to skin in anterior posterior and lateral dimensions and L1 to lesser trochanters craniocaudally.

- Sagittal bFFE/FIESTA/TrueFISP only if patient has had surgery or has a tumor or any known lesion.

- Ask the patient to empty urinary bladder before the examination and to breathe normally.

- Large subjects: increase voxel size to 1.5 to $2 \mathrm{~mm}$.

Table 4 Upper and lower extremity 3-T MRN protocol

\begin{tabular}{|c|c|c|c|c|c|c|c|}
\hline Sequence & Plane & Matrix & $\begin{array}{l}\text { Gap, } \\
\%\end{array}$ & Voxel size, $\mathrm{mm}$ & TR & $\mathrm{TE}$ & Comment \\
\hline $\begin{array}{l}\text { 2D T2 SPAIR/ } \\
\text { T2 Dixon }\end{array}$ & Axial & $256 \times 256$ & 10 & $3-4$ & 4,000 & $60-65$ & $\begin{array}{l}\text { Cover skin to skin to include area } \\
\text { of concern }\end{array}$ \\
\hline 2D T1 TSE & Axial & $256 \times 256$ & 10 & $3-4$ & 700 & $7-9$ & $\begin{array}{l}\text { Cover skin to skin to include area } \\
\text { of concern }\end{array}$ \\
\hline 3D SPAIR TSE & Coronal & $320 \times 320$ & 0 & Isotropic $1.0-1.1$ & 1,500 & 78 & $\begin{array}{l}\text { Cover } 10-15 \mathrm{~cm} \text { including the } \\
\text { area of interest }\end{array}$ \\
\hline 3D DW PSIF & Coronal & & 0 & Isotropic 0.9 & 12 & 2.5 & $\begin{array}{l}\text { Cover } 10-15 \mathrm{~cm} \text { including the } \\
\text { area of concern; b-value } 50-60\end{array}$ \\
\hline DTI & Axial & $128 \times 192$ & 0 & 4 & 10,000 & $60-70$ & $\begin{array}{l}\text { b-values of } 0 \text { and } 600,12 \text { direc- } \\
\text { tions. Perform around the } \\
\text { palpable or pain site. Keep FOV } \\
\text { under } 15 \mathrm{~cm} \text {. SPAIR fat saturation }\end{array}$ \\
\hline STIR (optional) & Axial & $256 \times 256$ & 10 & & 3000 & $25-35$ & If fat saturation fails on SPAIR \\
\hline $\begin{array}{l}\text { 3D T1 VIBE } \\
\text { (optional) }\end{array}$ & Axial & $320 \times 320$ & 0 & Isotropic 1.5 & 12 & 5 & Pre- and postcontrast if needed \\
\hline
\end{tabular}

Abbreviations: D, dimensional; DTI, diffusion tensor imaging; MIP, maximum intensity projection; PSIF, time-reversed FISP (fast imaging with steadystate precession); STIR, short tau inversion recovery; TE, time of echo; TR, time of repetition; TSE, turbo spin echo; VIBE, volumetric interpolated breath-hold examination.

- Upper extremity: Perform in superman position with extremity at the center of the field of view. Cover from skin to skin (not $>20 \%$ air space around the extremity). Use appropriate coil: wrist coil, flex coil for hand and forearm, etc.

- Lower extremity: Perform only one side at a time. Cover from skin to skin (not > 20\% air space around the extremity). Use appropriate coil: knee coil, flex coil for thigh, leg, foot, boot coil for ankle, etc. For dedicated ankle MRN, replace coronal fat-saturated PD with sagittal fat-saturated PD.

- Large subjects: Increase voxel size to 1.5 to $2 \mathrm{~mm}$. 
caliber without distal tapering are abnormal and indicative of neuropathy. Discontinuity of the nerve with end-bulbneuroma is indicative of a high-grade neural injury (Sunderland class IV and V).

The fascicular morphology has important diagnostic significance and should be identifiable on the axial images. Nerve thickness and image quality are key factors for this identification. Generally speaking, evaluation of the fascicular morphology is feasible in nerves $>3 \mathrm{~mm}$ thick. ${ }^{48}$ Fascicles should be examined regarding their uniformity, signal, size, and continuity. Enlarged, atrophied, nonuniform, or disrupted fascicles are pathologic. Comparison with the contralateral nerve (if imaged) or adjacent similar size nerves can be helpful for more precise interpretation.

SI is one of the most important features to be evaluated on MRN after suppressing adjacent perineural fat and vascular flow signal to obtain optimal SNR. The perineurium and outer epineurium demonstrate a thin low SI lining. As a rule, the SI of a peripheral nerve should be close to the adjacent skeletal muscle on all pulse sequences. ${ }^{34}$ High SI is seen on T2-weighted images at areas of neuropathy. This change in SI is usually evident within the first 24 hours following an insult. ${ }^{49,50}$ Nerve SI changes are maximum adjacent to the area of entrapment or injury that helps localize the site of insult. For instance, ulnar nerve hyperintensity is most pronounced at the cubital tunnel in cubital tunnel syndrome, and sciatic nerve signal alteration is most pronounced at the sciatic notch in piriformis syndrome. A longer extent of signal alteration is seen on the neuropathic side as compared with the contralateral normal nerve (e.g., in piriformis syndrome, a longer extent and more hyperintense signal is seen in the sciatic nerve as compared with minimal signal at the sciatic notch on the contralateral side). Diffuse signal or caliber changes in one or more regional nerves is indicative of a systemic neuropathy. That said, not all neural T2 hyperintensity is necessarily abnormal. Neural T2 hyperintensity can be found in asymptomatic subjects at the cubital and carpal tunnels in particular. In these situations, the degree and length of hyperintensity, the nerve caliber, and, whenever possible, comparison with the contralateral side should also be considered.
Nerves are generally wrapped in a fine fatty background referred to as the perineural fat. Obliteration of perineural fat planes by fibrosis is an indirect sign of entrapment or adhesive neuropathy secondary to a regional insult (e.g., in a failed back surgery syndrome, perineural scarring of the preganglionic segment with nerve entrapment and resultant downstream neuropathy or the femoral with or without sciatic nerves) (-Fig. 4). Other indirect signs of prior dural injury may be present such as dural diverticula, peridural iatrogenic fat patch, or pseudomeningocele apart from the regional scar tissue. In extremity nerve entrapment cases, perineural space-occupying lesions or nerve entrapment along the anatomical tunnels due to tight fascia, tenosynovitis, ganglion, accessory muscles, or other secondary cause can result in displacement of perineural fat planes and predispose to secondary changes in nerve course, caliber, and abnormal fascicular morphology and SI.

Use of contrast in routine MRN studies is controversial and differs from practice to practice. Some endorse its use; others argue that it provides little or no incremental value to the information obtained from noncontrast images. Normal peripheral nerves do not show appreciable enhancement after contrast administration due to the existence of a blood-nerve barrier. Enhancement could be physiologic as in dorsal nerve ganglia ${ }^{16}$ or indicate disruption of the blood-nerve barrier, a sign of neuropathy distal to the dorsal nerve root ganglion. Given the cost, increase in scan time, concerns regarding tissue accumulation of gadolinium-based contrast media, and its limited additive diagnostic value, the general consensus is against the routine use of contrast media in MRN studies. Contrast use is recommended if there is concern regarding infection, tumor, or atypical polyneuropathy. 2,34,51 Therefore, the supervising radiologist should consider each MRN case separately.

Muscle changes are of high clinical and diagnostic value in the setting of motor neuropathies and must always be taken into consideration. Muscle denervation change helps in more precise localization of the nerve lesion based on the anatomical distribution of the muscle changes. The neuropathy is always proximal to the denervation change in the muscle clinically or on MRN. Muscle changes can help determine the
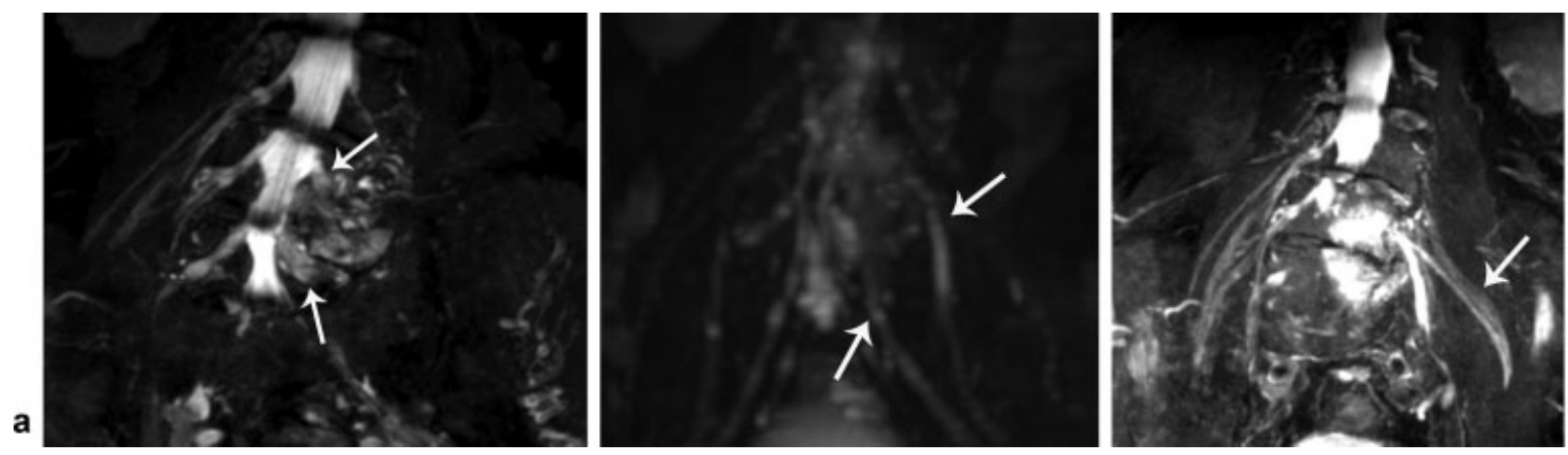

b, c

Fig. 4 A 75-year-old man presented with scoliosis and worsening pain in his left leg 4 months after posterior decompression. (a) Coronal 3D short tau inversion recovery and (b) DWI (b600) reconstructed maximum intensity projection images with a 14-mm slab thickness demonstrate increased signal intensity and abnormal thickening of the left L4 and L5 nerve roots (arrows, Sunderland class III) with significant perineural fibrosis/granulation as well as downstream sciatic (not shown) and (c) femoral neuropathy (arrow). The final diagnosis was postoperative perineural fibrosis with left L4 and L5 constriction. 
chronicity of the neuropathy with muscle edema indicative of a more acute injury (within a few days) and muscle fatty infiltration or atrophy with or without edema indicating a more chronic process. Muscle denervation edema or fatty infiltration is differentiated from an inflammatory myositis using key imaging signs such as diffuse involvement, lack of hemorrhage, or fascial/subcutaneous edema findings limited to the distribution of affected nerves and associated nerve lesions. Severe muscle atrophy is a poor prognostic indicator for nerve injury because even if nerve regeneration occurs, the muscle is weakened, and therefore a full functional recovery is unlikely. ${ }^{29}$ A serial decrease in muscle edema and nerve hyperintensity is correlated with nerve regeneration and subsequent functional improvement. ${ }^{49,52}$

\section{Tip 8: Become Familiar with the Key Pearls and Pitfalls of Imaging Diagnosis}

Knowledge of and attention to the most common diagnostic pearls and pitfalls will expedite the MRN learning process, as well as help generate accurate reports and aid patient management.

A frequent pitfall in interpretation occurs when it comes to nerve SI. Misinterpretation of an elevated nerve SI on fluidsensitive sequences is common, especially for less experienced eyes. This is even more common where nerves track along certain anatomical tunnels, such as the ulnar nerve or median nerve along the cubital and carpal tunnels, respectively. Mild increased SI of the ulnar nerve on T2-weighted images can be considered normal and should be correlated clinically. ${ }^{53}$ Conversely, some patients with clinical findings of cubital tunnel syndrome demonstrate only mild ulnar nerve signal changes at the tunnel. The same pitfall is seen when there is signal alteration on STIR images without a change in SI on fat-saturated T2-weighted images. Comparison with the contralateral asymptomatic nerve is very helpful although not always technically feasible in most cases in the upper extremities. A contrast-tonoise ratio method, where ulnar nerve SI is compared with background noise, may be helpful if doubt still exists. ${ }^{54}$

Therefore, experience and correlation with other MRN features of neuropathy such as distal longitudinal extension of signal hyperintensity, nerve enlargement, change in fascicular morphology, muscle denervation change, and not least correlation with clinical findings are important to avoid overrating these subtle changes. ${ }^{29,34,53,54}$ However, mild signal changes should not be overlooked because it may be the only sign of neuropathy. Knowledge of expected increased signal of nerve at certain sites due to magic angle artifacts is essential (e.g., the plexus nerves are bright at their curving points; the sciatic nerve at the sciatic notch, and the femoral nerve at its genu above the inguinal ligament, etc.).

Another important imaging feature to be aware of is the "triple B-sign" as described by Chhabra et al, and a variant described as the "bull's-eye sign" by Sneag et al orthogonal to the long axis of the nerve, where there is alternating brightblack-bright SI changes along the course of a nerve at the area of concern for injury or entrapment. This basically indicates severe focal neuropathy such as severe nerve entrapment, nerve transection, or severe degeneration/dehiscence of a nerve graft. ${ }^{29,48,55}$ Although isolated signal change can be associated with mild entrapment, proximal enlargement of nerve is a sign of moderate entrapment, and proximal and distal enlargement of the nerve is a sign of severe entrapment. In chronic entrapment cases, the SI may fade to near normal, although enlargement of the nerve with distal flattening at the site of entrapment will persist. In chronic neuropathies, such as DM, nerve signal may decrease on fat-suppressed T2-weighted images due to fascicular atrophy and increased epineurial fat. ${ }^{56}$

Another interpretation pitfall in nerve evaluation is the absence of perineural fat planes. Perineural fat is generally sparse or absent especially within anatomical tunnels and tight anatomical regions. Therefore, in its absence, it is hard to distinguish scar tissue from normal lack of perineural fat. Radiologists must avoid overestimating neuropathy based solely based on the absence of perineural fat, and correlation with anatomical location and other imaging features should be used.

Careful attention should be taken when protocoling MRN studies and deciding the FOV. Nerves are commonly enlarged with high SI proximal to the site of insult. However, distally, the nerves might depict normal SI and size, or even a mild decrease in diameter. Therefore, limited coverage of the FOV not including the proximal aspects of the nerve could lead to misinterpretation. Again, comparison with the contralateral normal nerve (if imaged) or the same size regional nerves, and paying attention to muscle denervation changes can be helpful. As a rule, the FOV must extend above the site of visible muscle denervation change.

Nerves may demonstrate anomalous course as a normal variant. Many of these alterations are asymptomatic, often bilateral, and therefore are of no clinical importance. However, some atypical courses are associated with entrapment/compressive neuropathies, such as bifid brachial nerve roots or an intramuscular course of the sciatic nerve. It is crucial to have a good knowledge of normal anatomy as well as being familiar with the common clinically important normal variants. ${ }^{47,57}$

MRN provides valuable information complementing other more diagnostic techniques, such as clinical examination and electrodiagnostic tests. Radiologists and clinicians should work as a team and relay all pieces of information in a timely fashion to get the most out of advanced diagnostic tests such as MRN. Many imaging pitfalls are related to lack of proper clinical information, EMG/NCV results, or miscommunication. This is especially important in inflammatory/ autoimmune neuropathies (Parsonage-Turner syndrome) or more diffuse and multifocal conditions. In these circumstances, neural thickening and altered signal are often diffuse and may be underrated by radiologists, especially if imaging is performed during the early, relatively asymptomatic period when findings are more subtle. ${ }^{56,58}$

\section{Tip 9: How and What to Report}

Important advantages of MRN over more traditional diagnostic techniques include its ability to assess neuromuscular anatomy precisely, localize neuropathy, show the extent and nature of neuromuscular abnormality or nerve injury, find 
organic lesions, and evaluate adjacent joint or tendon derangements that could predispose or contribute to traction neuropathy. These pieces of information put together with the clinical history and electrodiagnostic information can help better identify the cause of any neuropathy and target management. In addition, this information opens an array of other therapeutic advantages, such as for surgical planning, nerve blocks, and, if necessary, tissue sampling. Another advantage is that MRN is extremely sensitive with a high negative predictive value. Finding normal nerves in a case of suspected neuropathy can favor a psychological or psychosomatic etiology rather than an organic cause in a chronic pain patient.

The radiologist should comment on the degree of neuropathy based on the MRN findings. This is especially important prognostically and for care management. For instance, a Sunderland class IV and V injury needs surgical intervention, whereas milder neuropathies can be expected to improve with conservative management. The Seddon and Sunderland classification of neuropathy is based on various layers of neural tissue involvement. This classification can be used for MRN interpretation and reporting; however, not all delicate neural tissue layers can be individually evaluated even on highresolution MRN studies. Therefore, the following grading system can alternatively be used in addition to the Seddon and Sunderland grading, especially for less experienced readers. This grading system is capable of conveying all the required clinically important information, is more practical, and easier to communicate MRN findings.

- Low-grade (stretch) injury (equivalent to Sunderland class I or neuropraxia based on Seddon): nerve-in-continuity with increased T2 SI without focal or diffuse enlargement
- Moderate-grade injury (equivalent to Sunderland class II/ III or axonotmesis based on Seddon): nerve-in-continuity with diffuse nerve enlargement (more than the size of the contralateral nerve or adjacent vessel) with increased T2 SI with or without fascicular abnormalities

- High-grade injury (equivalent to Sunderland IV): NIC with abrupt change in nerve caliber, effacement of or heterogeneous fascicular morphology, and increased T2 SI (-Figs. 5 and 6)

- Severe injury: nerve transection or nerve root avulsion with or without end-bulb-neuroma formation (equivalent to Sunderland class $\mathrm{V}$ or neurotmesis based on Seddon)

Radiologists must try to follow a specific search pattern and consider using a structured template with a checklist approach while interpreting MRN studies. Having a specific step-by-step search algorithm probably reduces the likelihood of overlooking subtler but often critically important findings, such as anatomical variations, obliteration of perineural fat planes, and muscle denervation changes. Use of proper and accurate terminology is crucial in describing and communicating nerve findings. Excessive use of "radiology language" is confusing for clinicians, and information may get overlooked during report interpretation. Adherence of both radiologists and clinicians to a recognized classification system is crucial in this regard to avoid preventable diagnostic pitfalls. ${ }^{29,59}$ For example, myopathy in neurology literature refers to nonneurogenic muscle pathology. Using the term muscle denervation change instead of myopathy helps multidisciplinary communication. The nerve should not just be called bright. Interpretation must be more specific. The patient history and clinical findings need to
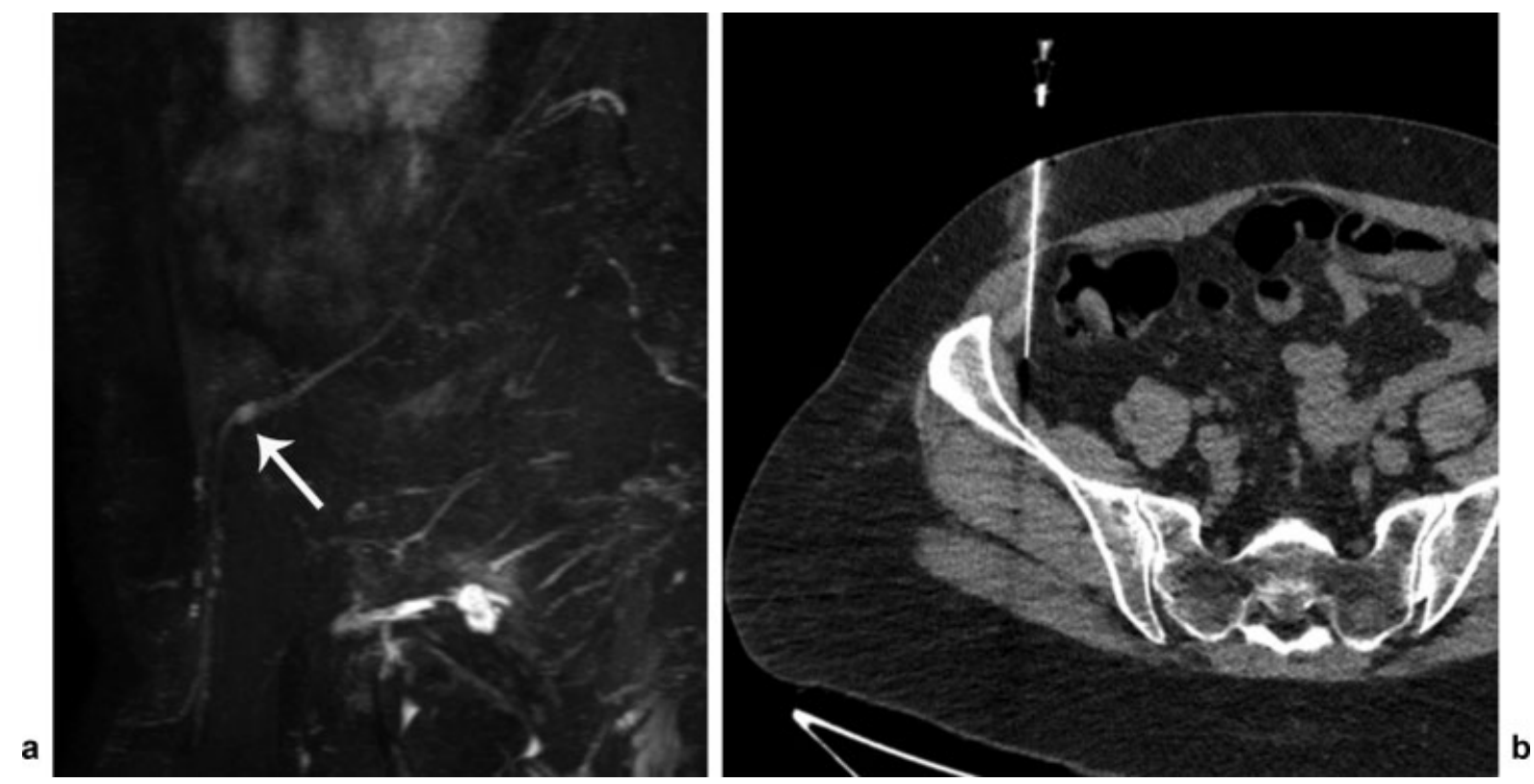

Fig. 5 A 68-year-old woman with pain and paresthesia along the right lateral thigh. Lumbar plexus MR neurography was performed for suspected meralgia paresthetica. (a) Maximum intensity projection coronal oblique 3D short tau inversion recovery image with 14 -mm slice thickness depicts increased thickness and signal intensity of the right lateral femoral cutaneous nerve (LFCN). There is a small 4-mm neuroma-incontinuity (NIC) along the right LFCN (arrow) at the level of the inguinal ligament. (b) Intraprocedural axial computed tomography image shows LFCN block proximal to the area affected by the NIC. 
a
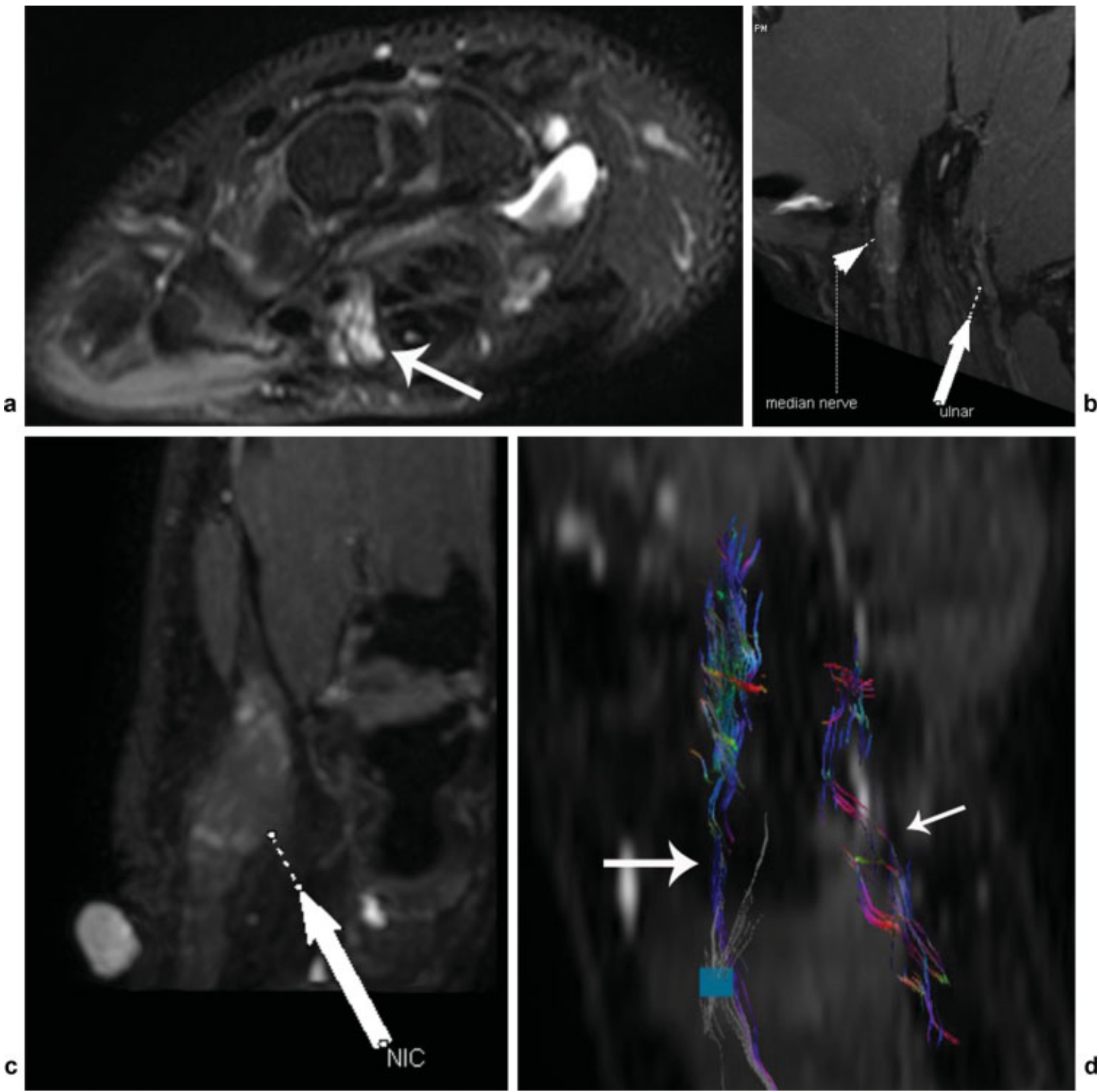

Fig. 6 A 37-year-old man with a history of carpal tunnel release presented with numbness and tingling along the median nerve distribution. (a) Axial T2 SPAIR image shows marked thickening and bright SI on the median nerve (arrow) at the carpal tunnel. (b) Coronal and (c) sagittal MIP 3D PSIF images depict a large neuroma-in-continuity (NIC) measuring $2.5 \mathrm{~cm}$ along the median nerve. (d) Diffusion tensor imaging tractography map demonstrates marked disruption of fascicles (markedly reduced relative to the size of the nerve) at the site of NIC (large arrow) consistent with axonal degeneration and demyelination. Fractional anisotropy (FA) and apparent diffusion coefficient (ADC) measured 0.45 and $1.31 \times 10^{-3} \mathrm{~mm}^{2} / \mathrm{s}$, respectively. Notice normal ulnar nerve (small arrow, $\mathrm{FA}=0.6, \mathrm{ADC}=1.10 \times 10^{-3} \mathrm{~mm}^{2} / \mathrm{s}$ ).

be reviewed, as well as a quite specific diagnosis suggested based on all available information and included as part of the final impression in the report.

\section{Tip 10: How to Participate in Multidisciplinary Care of Neuropathy Patients}

Radiologists are, in most cases, consultants in the multidisciplinary team. On a personal note, we at UT Southwestern are one of the rare sites in the world with a dedicated musculoskeletal patient care clinic where patients with musculoskeletal disorders and neuropathy are seen by radiologists, imaged, and intervened. Whichever model exists in your center, it is important to be part of the multidisciplinary care of the patients with formation of disease-oriented teams in this era of patientcentered care. Regular communication of the findings and continuous learning from your other clinical colleagues (plastic surgeons, peripheral nerve surgeon, neurologists, fellow radiologists, etc.) is not only helpful for proper patient care but also essential for personal and professional growth. The treatment decisions about nerve injections, medications, and surgery are best made with multiple teams working together facilitating cost-effective care. The knowledge gained can be distributed in the form of case series and scientific reports to less experienced readers in other centers. ${ }^{60,61}$ 
To conclude, MRN has rapidly translated into current clinical practice. We have outlined what we consider to be the most important tips to help set up a successful MRN practice.

\section{Financial Disclosure}

Avneesh Chhabra is a consultant for ICON Medical and receives royalties from Jaypee and Wolters.

\section{Conflicts of Interest}

None declared.

\section{References}

1 Filler AG, Kliot M, Howe FA, et al. Application of magnetic resonance neurography in the evaluation of patients with peripheral nerve pathology. J Neurosurg 1996;85(02):299-309

2 Manoliu A, Ho M, Nanz D, et al. MR neurographic orthopantomogram: Ultrashort echo-time imaging of mandibular bone and teeth complemented with high-resolution morphological and functional MR neurography. J Magn Reson Imaging 2016;44 (02):393-400

3 Viallon M, Vargas MI, Jlassi H, Lövblad KO, Delavelle J. Highresolution and functional magnetic resonance imaging of the brachial plexus using an isotropic 3D T2 STIR (short term inversion recovery) SPACE sequence and diffusion tensor imaging. Eur Radiol 2008;18(05):1018-1023

4 Linda DD, Harish S, Stewart BG, Finlay K, Parasu N, Rebello RP. Multimodality imaging of peripheral neuropathies of the upper limb and brachial plexus. Radiographics 2010;30(05):1373-1400

5 Andreisek G, Burg D, Studer A, Weishaupt D. Upper extremity peripheral neuropathies: role and impact of MR imaging on patient management. Eur Radiol 2008;18(09):1953-1961

6 Bashir WA, Connell DA. Imaging of entrapment and compressive neuropathies. Semin Musculoskelet Radiol 2008;12(02):170-181

7 Chalian M, Behzadi AH, Williams EH, Shores JT, Chhabra A. Highresolution magnetic resonance neurography in upper extremity neuropathy. Neuroimaging Clin N Am 2014;24(01):109-125

8 Subhawong TK, Wang KC, Thawait SK, et al. High resolution imaging of tunnels by magnetic resonance neurography. Skeletal Radiol 2012;41(01):15-31

9 Martinoli C, Bianchi S, Pugliese F, et al. Sonography of entrapment neuropathies in the upper limb (wrist excluded). J Clin Ultrasound 2004;32(09):438-450

10 Salvalaggio A, Cacciavillani M, Coraci D, et al. Nerve ultrasound and 3D-MR neurography suggestive of intraneural perineurioma. Neurology 2016;86(12):1169-1170

11 Brown JM, Yablon CM, Morag Y, Brandon CJ, Jacobson JA. US of the peripheral nerves of the upper extremity: a landmark approach. Radiographics 2016;36(02):452-463

12 Ohana M, Moser T, Moussaouï A, et al. Current and future imaging of the peripheral nervous system. Diagn Interv Imaging 2014;95 (01):17-26

13 Chhabra A, Belzberg AJ, Rosson GD, et al. Impact of high resolution 3 Tesla MR neurography (MRN) on diagnostic thinking and therapeutic patient management. Eur Radiol 2016;26(05):1235-1244

14 Fisher S, Wadhwa V, Manthuruthil C, Cheng J, Chhabra A. Clinical impact of magnetic resonance neurography in patients with brachial plexus neuropathies. Br J Radiol 2016;89(1067):20160503

15 Andreisek G, Bolog NV. Getting started with magnetic resonance neurography. Semin Musculoskelet Radiol 2018;22(03):334-343

16 Filler AG, Maravilla KR, Tsuruda JS. MR neurography and muscle MR imaging for image diagnosis of disorders affecting the peripheral nerves and musculature. Neurol Clin 2004;22(03): 643-682, vi-vii
17 Kierszenbaum AL, Tres L. Histology and Cell Biology Pageburst E-book on Vitalsource Retail Access Card: An Introduction to Pathology. 4th ed. Philadelphia, PA: Saunders; 2015

18 Ratner S, Khwaja R, Zhang L, et al. Sciatic neurosteatosis: relationship with age, gender, obesity and height. Eur Radiol 2018;28(04): 1673-1680

19 Eastlack J, Tenorio L, Wadhwa V, Scott K, Starr A, Chhabra A. Sciatic neuromuscular variants on MR neurography: frequency study and interobserver performance. Br J Radiol 2017;90 (1079):20170116

20 Jabaley ME, Wallace WH, Heckler FR. Internal topography of major nerves of the forearm and hand: a current view. J Hand Surg Am 1980;5(01):1-18

21 Thawait SK, Chaudhry V, Thawait GK, et al. High-resolution MR neurography of diffuse peripheral nerve lesions. AJNR Am J Neuroradiol 2011;32(08):1365-1372

22 Zuniga JR, Mistry C, Tikhonov I, Dessouky R, Chhabra A. Magnetic resonance neurography of traumatic and nontraumatic peripheral trigeminal neuropathies. J Oral Maxillofac Surg 2018;76(04): 725-736

23 Seddon H. Three types of nerve injury. Brain 1943;66:237-288

24 Sunderland S. A classification of peripheral nerve injuries producing loss of function. Brain 1951;74(04):491-516

25 Mackinnon S, Dellon A. Surgery of the Peripheral Nerve. New York, NY: Thieme; 1988:74-78

26 Dyck PJ. Invited review: limitations in predicting pathologic abnormality of nerves from the EMG examination. Muscle Nerve 1990;13(05):371-375

27 Belzberg AJ. Acute nerve injuries. In: Rengachary SS, Ellenbogen RG, eds. Principles of Neurosurgery. 2nd ed. Philadelphia, PA: Elsevier Mosby; 2005:387-395

28 Zhang H, Xiao B, Zou T. Clinical application of magnetic resonance neurography in peripheral nerve disorders. Neurosci Bull 2006; 22(06):361-367

29 Andreisek G, Chhabra A. MR neurography: pitfalls in imaging and interpretation. Semin Musculoskelet Radiol 2015;19(02):94-102

30 Andreisek G, White LM, Kassner A, Sussman MS. Evaluation of diffusion tensor imaging and fiber tractography of the median nerve: preliminary results on intrasubject variability and precision of measurements. AJR Am J Roentgenol 2010;194(01):W65-W72

31 Guggenberger R, Nanz D, Puippe G, et al. Diffusion tensor imaging of the median nerve: intra-, inter-reader agreement, and agreement between two software packages. Skeletal Radiol 2012;41 (08):971-980

32 Guggenberger R, Eppenberger P, Markovic D, et al. MR neurography of the median nerve at 3.0T: optimization of diffusion tensor imaging and fiber tractography. Eur J Radiol 2012;81(07): e775-e782

33 Guggenberger R, Nanz D, Bussmann L, et al. Diffusion tensor imaging of the median nerve at 3.0 T using different MR scanners: agreement of FA and ADC measurements. Eur J Radiol 2013;82 (10):e590-e596

34 Chhabra A, Lee PP, Bizzell C, Soldatos T. 3 Tesla MR neurographytechnique, interpretation, and pitfalls. Skeletal Radiol 2011;40 (10):1249-1260

35 Chhabra A, Flammang A, Padua A Jr, Carrino JA, Andreisek G. Magnetic resonance neurography: technical considerations. Neuroimaging Clin N Am 2014;24(01):67-78

36 Menezes CM, de Andrade LM, Herrero Cda S, et al. Diffusionweighted magnetic resonance (DW-MR) neurography of the lumbar plexus in the preoperative planning of lateral access lumbar surgery. Eur Spine J 2015;24(04):817-826

37 Chhabra A, Faridian-Aragh N, Chalian M, et al. High-resolution 3-T MR neurography of peroneal neuropathy. Skeletal Radiol 2012;41 (03):257-271

38 Wang X, Harrison C, Mariappan YK, et al. MR neurography of brachial plexus at $3.0 \mathrm{~T}$ with robust fat and blood suppression. Radiology 2017;283(02):538-546 
39 Ahlawat S, Stern SE, Belzberg AJ, Fritz J. High-resolution metal artifact reduction MR imaging of the lumbosacral plexus in patients with metallic implants. Skeletal Radiol 2017;46(07):897-908

40 Mürtz P, Kaschner M, Lakghomi A, et al. Diffusion-weighted MR neurography of the brachial and lumbosacral plexus: 3.0 T versus 1.5 T imaging. Eur J Radiol 2015;84(04):696-702

41 Eppenberger P, Andreisek G, Chhabra A. Magnetic resonance neurography: diffusion tensor imaging and future directions. Neuroimaging Clin N Am 2014;24(01):245-256

42 Yoneyama M, Takahara T, Kwee TC, Nakamura M, Tabuchi T. Rapid high resolution MR neurography with a diffusion-weighted prepulse. Magn Reson Med Sci 2013;12(02):111-119

43 Chhabra A, Carrino JA, Farahani SJ, et al. Whole-body MR neurography: Prospective feasibility study in polyneuropathy and CharcotMarie-Tooth disease. J Magn Reson Imaging 2016;44(06):1513-1521

44 Vaeggemose M, Vaeth S, Pham M, et al. Magnetic resonance neurography and diffusion tensor imaging of the peripheral nerves in patients with Charcot-Marie-Tooth type 1A. Muscle Nerve 2017;56(06):E78-E84

45 Vaeggemose M, Pham M, Ringgaard S, et al. Magnetic resonance neurography visualizes abnormalities in sciatic and tibial nerves in patients with type 1 diabetes and neuropathy. Diabetes 2017; 66(07):1779-1788

46 Wu C, Wang G, Zhao Y, et al. Assessment of tibial and common peroneal nerves in diabetic peripheral neuropathy by diffusion tensor imaging: a case control study. Eur Radiol 2017;27(08): 3523-3531

47 Soldatos T, Andreisek G, Thawait GK, et al. High-resolution 3-T MR neurography of the lumbosacral plexus. Radiographics 2013;33 (04):967-987

48 Chhabra A, Madhuranthakam AJ, Andreisek G. Magnetic resonance neurography: current perspectives and literature review. Eur Radiol 2018;28(02):698-707

49 Bendszus M, Wessig C, Solymosi L, Reiners K, Koltzenburg M. MRI of peripheral nerve degeneration and regeneration: correlation with electrophysiology and histology. Exp Neurol 2004;188(01):171-177

50 van Ouwerkerk WJR, Strijers RLM, Barkhof F, Umans U, Vandertop WP. Detection of root avulsion in the dominant C7 obstetric brachial plexus lesion: experience with three-dimensional con- structive interference in steady-state magnetic resonance imaging and electrophysiology. Neurosurgery 2005;57(05):930-940; discussion 930-940

51 Chabbra A, Andreisek G. Magnetic Resonance Neurography. London, UK: Jaypee; 2012

52 Viddeleer AR, Sijens PE, van Ooijen PMA, et al. Quantitative STIR of muscle for monitoring nerve regeneration. J Magn Reson Imaging 2016;44(02):401-410

53 Husarik DB, Saupe N, Pfirrmann CWA, Jost B, Hodler J, Zanetti M. Elbow nerves: MR findings in 60 asymptomatic subjects-normal anatomy, variants, and pitfalls. Radiology 2009;252(01):148-156

54 Bäumer P, Dombert T, Staub F, et al. Ulnar neuropathy at the elbow: MR neurography-nerve T2 signal increase and caliber. Radiology 2011;260(01):199-206

55 Sneag DB, Saltzman EB, Meister DW, Feinberg JH, Lee SK, Wolfe SW. MRI bullseye sign: an indicator of peripheral nerve constriction in Parsonage-Turner syndrome. Muscle Nerve 2017;56(01): 99-106

56 Thakkar RS, Del Grande F, Thawait GK, Andreisek G, Carrino JA, Chhabra A. Spectrum of high-resolution MRI findings in diabetic neuropathy. AJR Am J Roentgenol 2012;199(02):407-412

57 Chhabra A, Farahani SJ, Thawait GK, Wadhwa V, Belzberg AJ, Carrino JA. Incremental value of magnetic resonance neurography of lumbosacral plexus over non-contributory lumbar spine magnetic resonance imaging in radiculopathy: a prospective study. World J Radiol 2016;8(01):109-116

58 Wolf M, Bäumer P, Pedro M, et al. Sciatic nerve injury related to hip replacement surgery: imaging detection by MR neurography despite susceptibility artifacts. PLoS One 2014;9(02):e89154

59 Chhabra A, Ahlawat S, Belzberg A, Andreseik G. Peripheral nerve injury grading simplified on MR neurography: as referenced to Seddon and Sunderland classifications. Indian J Radiol Imaging 2014;24(03):217-224

60 Dessouky R, Khaleel M, Khalifa DN, Tantawy HI, Chhabra A. Magnetic resonance neurography of the lumbosacral plexus in failed back surgery syndrome. Spine 2018;43(12):839-847

61 Dessouky R, Xi Y, Scott KM, et al. Magnetic resonance neurography in chronic lumbosacral and pelvic pain: diagnostic and management impact-institutional audit. World Neurosurg 2018;114:e77-e113 